\title{
ESTADO NUTRICIONAL E FATORES ASSOCIADOS EM IDOSOS: EVIDÊNCIAS COM BASE EM INQUÉRITO TELEFÔNICO
}

\section{Nutritional status and associated factors in the elderly: evidence based on telephone survey \\ Estado nutricional y factores asociados en ancianos: evidencias basadas en encuesta telefónica}

\author{
Célia Cristina Diogo Ferreira \\ Universidade Federal do Rio de Janeiro - UFRJ - Macaé (RJ) - Brasil
}

\section{Gina Torres Rego Monteiro}

Escola Nacional de Saúde Pública Sérgio Arouca - ENSP/Fiocruz - Rio de Janeiro (RJ) - Brasil

Taynãna César Simões

Centro de Pesquisa René Rachou - CPqRR /Fiocruz - Belo Horizonte (MG) - Brasil

\section{RESUMO}

Objetivo: Avaliar o estado nutricional e fatores associados em idosos. Métodos: Trata-se de um estudo transversal, realizado entre junho e outubro de 2016, com 720 idosos. Utilizaram-se informações demográficas, socioeconômicas, comportamentais e de estado de saúde, de indivíduos com idade maior ou igual a 60 anos, provenientes do Sistema de Vigilância de Fatores de Risco e Proteção para Doenças Crônicas por inquérito telefônico (VIGITEL), no ano de 2013. Foram ajustadas as medidas de associação para sobrepeso e obesidade, de acordo com variáveis estudadas, segundo modelo logístico binomial. Resultados: A maioria dos participantes era do sexo feminino $(59,7 \%, \mathrm{n}=430)$, com escolaridade até o ensino fundamental $(70,5 \%, \mathrm{n}=508)$ e não trabalhavam $(75,2 \%, \mathrm{n}=541)$. A prevalência de excesso de peso e de obesidade nos idosos foi de 57,5\% ( $\mathrm{n}=414)$ e 19,9\% ( $\mathrm{n}=143)$, respectivamente. Substituíam refeições principais por lanche (cinco ou mais vezes na semana) $33,8 \%(n=243)$ dos idosos. Referiram pressão alta 62,8\% $(n=452)$ dos idosos, $21,2 \%(n=153)$ eram diabéticos, 35,4\% ( $=255)$ apresentavam colesterol alto e $35,4 \%(n=255)$ tinham dislipidemia. Ser hipertenso e ter dislipidemia aumentavam a chance de ter excesso de peso. $\mathrm{O}$ aumento da idade diminuiu a chance de ter excesso de peso. A obesidade foi positivamente associada à hipertensão arterial, à dislipidemia e ao sexo feminino. Conclusão: Observou-se alto percentual de excesso de peso e obesidade, sendo os idosos com pressão alta, dislipidemia e hábito de substituir as refeições principais por lanche os com maiores chances de apresentar excesso de peso e obesidade.

Descritores: Sobrepeso; Obesidade; Idosos; Inquéritos Epidemiológicos.

\section{ABSTRACT}

Objective: To evaluate the nutritional status and associated factors in the elderly. Methods: This is a cross-sectional study, carried out between June and October 2016, with 720 elderly individuals. It used demographic, socioeconomic, behavioral and health status information on individuals aged 60 years or older, originating from the system Surveillance of Risk and Protective Factors for Chronic Diseases through Telephone Survey (VIGITEL), in the year 2013. The measures of association for overweight and obesity were adjusted according to some variables studied, on the binomial logistic model. Results: Most of the participants were women (59.7\%, $n=430)$, with education until middle school $(70.5 \%, n=508)$, and did not work $(75.2 \%, n=541)$. The prevalence of excess weight and obesity in the elderly was $57.5 \%(n=414)$ and $19.9 \%$ (n=143), respectively. Main meals were replaced with snacks (five or more times per week) by 33.8\% ( $n=243)$ of the elderly. High blood pressure was reported by $62.8 \%(n=452)$ of the elderly; $21.2 \%(n=153)$ were diabetic, $35.4 \%(n=255)$ had high cholesterol, and $35.4 \%$ $(n=255)$ had dyslipidemia. Being hypertensive and having dyslipidemia increased the likelihood of being overweight. Increasing age reduced the likelihood of being overweight. Obesity was positively associated with hypertension, dyslipidemia and female gender. Conclusion: A high percentage of excess weight and obesity was observed, and elderly individuals with high blood pressure, dyslipidemia and the habit of replacing main meals with snacks were more likely to be overweight and obese.

Descriptors: Overweight; Obesity; Aged; Health Surveys. 


\section{RESUMEN}

Objetivo: Evaluar el estado nutricional y los factores asociados en ancianos. Métodos: Se trata de un estudio transversal realizado entre junio y octubre de 2016 con 720 ancianos. Se utilizaron las informaciones demográficas, socioeconómicas, de conductas y del estado de salud de individuos con 60 años de edad o más provenientes del Sistema de Vigilancia de Factores de Riesgo y Protección para las Enfermedades Crónicas a través de encuesta telefónica (VIGITEL) en al año de 2013. Se ajustaron las medidas de asociación para el sobrepeso y la obesidad según las variables estudiadas del modelo logístico binomial. Resultados: La mayoría de los participantes eran del sexo femenino $(59,7 \%, n=430)$ con máxima escolaridad de la educación primaria $(70,5 \%, n=508)$ y sin trabajo $(75,2 \%, n=541)$. La prevalencia del exceso de peso y de obesidad en los ancianos ha sido del 57,5\% (n=414) y 19,9\% $(n=143)$, respectivamente. El 33,8\% $(n=243)$ de los ancianos sustituian las comidas principales por meriendas (cinco o más veces a la semana). El 62,8\% (n=452) de los ancianos refirieron presión arterial alta, el 21,2\% (n=153) eran diabéticos, el 35,4\% (n=255) presentaban el colesterol alto y el 35,4\% ( $n=255)$ tenían dislipidemia. El hecho de ser hipertenso y tener dislipidemia aumentaba la oportunidad de tener exceso de peso. El aumento de la edad ha disminuido la oportunidad de tener exceso de peso. La obesidad estuvo asociada de manera positiva a la hipertensión arterial, la dislipidemia y al sexo femenino. Conclusión: Se observó un elevado porcentual de exceso de peso y obesidad y los ancianos con presión alta, dislipidemia y con la costumbre de substituir las principales comidas por meriendas son aquellos con más oportunidades de presentar exceso de peso y obesidad.

Descriptores: Sobrepeso; Obesidad; Anciano; Encuestas Epidemiológicas.

\section{INTRODUÇÃO}

A redução das taxas de fecundidade, diminuição da mortalidade e consequente aumento da expectativa de vida caracterizam o processo de reestruturação demográfica em que a população mundial se encontra. Isso se reflete em um decréscimo na proporção de crianças e jovens e um aumento na população de adultos e idosos. Esse último grupo é, atualmente, um contingente expressivo e de crescente importância na sociedade brasileira, acompanhado da necessidade de uma série de exigências e demandas em termos de políticas públicas de saúde( ${ }^{(1,2)}$.

O estado nutricional é um importante marcador de saúde nesses indivíduos, pois durante o envelhecimento, o organismo do idoso apresenta alterações fisiológicas que podem influenciar sua nutrição e, consequentemente, seu estado de saúde geral. Fatores como limitações financeiras, presença de enfermidades, uso de medicamentos, incapacidade física para o preparo dos alimentos e isolamento social podem interferir no acesso e seleção de alimentos e contribuir para o desenvolvimento de distúrbios nutricionais ${ }^{(3)}$.

Embora a prevalência de sobrepeso e obesidade em idosos brasileiros seja considerada alta (41,9\% no sexo feminino e $31,6 \%$ no masculino) ${ }^{(4)}$, esses indivíduos apresentam elevada inadequação na ingestão de nutrientes protetores contra doenças crônicas, tais como vitaminas e minerais antioxidantes, assim como gorduras insaturadas ${ }^{(5)}$. Inquéritos dietéticos telefônicos fornecem estimativas aproximadas de consumo alimentar, com investimento financeiro relativamente pequeno e menor tempo, possibilitando entender melhor as tendências no perfil de consumo alimentar e nutricional da população idosa brasileira ${ }^{(6)}$.

O sistema de Vigilância de Fatores de Risco e Proteção para Doenças Crônicas por Inquérito Telefônico (VIGITEL) foi implantado em 2006 em 27 capitais do Brasil. Seus resultados vêm propiciando melhoria na qualidade das informações epidemiológicas, assim como ampliação do conhecimento sobre as Doenças Crônicas Não Transmissíveis (DCNT) no país, juntamente com outros inquéritos nacionais ${ }^{(7,8)}$.

Com o aumento populacional dos idosos brasileiros, eleva-se a necessidade da investigação de fatores determinantes relacionados à saúde e ao estado nutricional para que as propostas de intervenção de saúde possam ser implementadas e causem impacto na qualidade de vida desse grupo. Nesse contexto, o presente artigo objetivou avaliar o estado nutricional e fatores associados em idosos.

\section{MÉTODOS}

Trata-se de estudo transversal e analítico, realizado entre junho e outubro de 2016, cujos dados foram obtidos a partir do inquérito telefônico VIGITEL, realizado no ano de $2013^{(9)}$. A população avaliada foi composta pelos indivíduos sorteados nesse inquérito, com idade maior ou igual a 60 anos de idade, residentes na cidade do Rio de Janeiro, Brasil, e com totalidade de informações para as variáveis de interesse.

A amostra analisada compreendeu 720 idosos (representativo do total de 1.679 .837 idosos na população da cidade do Rio de Janeiro). O IBGE estimou, no ano considerado, que 13\% da população era composta por idosos na cidade do Rio de Janeiro, portanto, a amostra do VIGITEL pode ser considerada representativa da população de idosos ${ }^{(9)}$. Apenas quatro indivíduos foram excluídos por não apresentarem resposta para as variáveis de interesse do presente estudo.

Todas as medidas de frequência e análises estatísticas levaram em consideração os pesos amostrais atribuídos a cada indivíduo entrevistado, contemplando o procedimento de amostragem complexa do inquérito. Esses pesos, além de corrigirem 
a maior chance de indivíduos de domicílios com mais de uma linha telefônica fossem selecionados para a amostra, também corrigiam a menor chance que indivíduos de domicílios habitados por mais pessoas tiveram de ser selecionados, fornecendo estimativas confiáveis.

As variáveis de desfecho foram obtidas a partir do índice de massa corporal (IMC) e classificadas em excesso de peso (Sim $\left.=\mathrm{IMC} \geq 25 \mathrm{Kg} / \mathrm{m}^{2}\right)$ e obesidade $\left(\mathrm{Sim}=\mathrm{IMC} \geq 30 \mathrm{Kg} / \mathrm{m}^{2}\right)$, de acordo com os critérios adotados pelo VIGITEL ${ }^{(9)}$. Avaliaram-se os possíveis determinantes, separadamente, para cada desfecho de interesse, a partir de associações com as variáveis categorizadas segundo o VIGITEL. Em relação às variáveis demográficas e socioeconômicas, utilizou-se idade, sexo, ter relação conjugal (Sim: casado legalmente ou em união estável há mais de seis meses; Não: solteiro, viúvo, separado ou divorciado), raça/cor (branca, não branca), escolaridade (analfabeto/não sabe/não quis responder, até fundamental, até ensino médio, superior ou mais), ter plano de saúde, ter trabalho.

Para avaliação do estado de saúde coletaram-se as variáveis de autoavaliação do estado de saúde, ter pressão alta, diabetes, colesterol alto ou dislipidemia. No tocante às comportamentais foram consideradas: consumo de cinco ou mais porções de hortaliças e/ou frutas na semana, consumo regular de doce ( 5 vezes ou mais na semana), hábito de substituir as refeições principais por lanche ( 5 vezes ou mais vezes por semana), consumo de leite integral, consumo alto ou muito alto de sal, realização suficiente de atividade física ${ }^{(10)}$, alto consumo de álcool, fumante diário (Sim; Não).

Realizou-se a análise descritiva das variáveis de interesse ajustando as medidas sumárias pelos pesos amostrais. As análises bivariadas entre variáveis categóricas foram avaliadas pelo teste qui-quadrado com correção de Rao Scott, e entre categorias pelo teste entre proporções, considerando os pesos amostrais ${ }^{(11)}$. Embora os dados sejam provenientes de um estudo seccional, a partir do qual se obtém medidas de prevalência, optou-se por detectar apenas associações e direções das mesmas, utilizando modelos de regressão logística, por meio da razão de chances, não atentando às magnitudes de tais medidas, possivelmente superestimadas $^{(12)}$, ajustando-se modelos separados para os dois desfechos de interesse.

No processo de modelagem, variáveis significativas nos modelos univariados foram sendo adicionadas aos modelos múltiplos em ordem decrescente de significância ( $\mathrm{p}$-valor $<0,20)$. As associações entre variáveis explicativas também foram avaliadas, a fim de evitar multicolinearidade. A inclusão de variáveis foi testada a cada passo pelo teste de razão de verossimilhança ${ }^{(13)}$. A posterior inclusão de variáveis não significativas na análise univariada também recebeu avaliação. Além disso, possíveis termos de interação entre as variáveis que permaneceram no modelo múltiplo foram testados. A contribuição individual dos efeitos no modelo múltiplo foi avaliada ao nível de $10 \%$ de significância, pelo teste de Wald, aliado à significância prática.

Todas as análises ocorreram no software estatístico R, utilizando o pacote survey, fornecendo estimativas ponderadas confiáveis $^{(14)}$. Por ser uma amostra complexa, os resultados serão apresentadas como proporções.

\section{RESULTADOS}

Do total de 720 idosos avaliados, 57,5\% (n=414) apresentaram excesso de peso e 19,9\% (n=143), obesidade. Verificouse uma amostra composta, em sua maioria, pelo sexo feminino $(59,7 \%, \mathrm{n}=430)$, com escolaridade até o ensino fundamental $(70,5 \%, n=508)$ e que não trabalhavam $(75,2 \%, n=541)$ (Tabela I).

Quanto às variáveis comportamentais: a maioria dos idosos amostrados consumia cinco ou mais porções de hortaliças $(57,1 \%, n=411)$ ou frutas $(73,1 \%, n=526)$ por semana; $15,4 \%(n=111)$ faziam consumo regular de doce (cinco ou mais vezes na semana); $33,8 \%(\mathrm{n}=243)$ substituíam as refeições principais por lanche com regularidade (cinco ou mais vezes por semana); $43,1 \%(n=310)$ consumiam leite integral e 5,1\% $(n=37)$ faziam alto ou muito alto consumo de sal. Quanto às variáveis comportamentais, 8,3\% $(n=60)$ apresentavam alto consumo de álcool e 7,2\% $(n=52)$ eram fumantes (Tabela I).

Em relação ao estado de saúde, apenas $8,6 \%(\mathrm{n}=62)$ avaliaram sua condição de saúde como ruim ou muito ruim. Além disso, 62,8\% $(\mathrm{n}=452)$ referiram pressão alta; 21,2\% $(\mathrm{n}=153)$ eram diabéticos; 35,4\% $(\mathrm{n}=255)$ apresentavam colesterol alto, e $35,4 \%(n=255)$ tinham dislipidemia. A idade média nos idosos com excesso de peso foi de 69,8 e de 68,5 anos naqueles com obesidade (Tabela I). 
Tabela I - Distribuição das porcentagens e análise bivariada do excesso de peso, obesidade e variáveis demográficas, socioeconômicas, comportamentais, e de estado de saúde, em idosos da cidade do Rio de Janeiro, VIGITEL 2013 , Brasil.

\begin{tabular}{|c|c|c|c|c|c|c|c|c|}
\hline \multirow{2}{*}{ Variáveis } & \multirow[b]{2}{*}{ Categorias } & \multirow{2}{*}{$\begin{array}{c}\text { Porcentagens } \\
\% \\
\end{array}$} & \multicolumn{2}{|c|}{ Excesso de Peso } & \multicolumn{4}{|c|}{ Obesidade } \\
\hline & & & $\begin{array}{l}\text { Sim } \\
(\%)\end{array}$ & $\begin{array}{l}\text { Não } \\
\text { (\%) }\end{array}$ & p-valor & $\begin{array}{l}\text { Sim } \\
(\%)\end{array}$ & $\begin{array}{l}\text { Não } \\
\text { (\%) }\end{array}$ & p-valor \\
\hline \multicolumn{9}{|c|}{ Demográficas } \\
\hline \multicolumn{9}{|l|}{ Sexo } \\
\hline & Masculino & 40,3 & 54,1 & 45,9 & 0,2324 & 14,9 & 85,1 & 0,0527 \\
\hline & Feminino & 59,7 & 59,8 & 40,2 & & 23,3 & 76,7 & \\
\hline \multicolumn{9}{|c|}{ Relação Conjugal } \\
\hline & Sim & 53,9 & 60,5 & 39,5 & 0,1535 & 21,2 & 78,8 & 0,4488 \\
\hline & Não & 46,1 & 54,0 & 46,0 & & 18,3 & 81,7 & \\
\hline \multicolumn{9}{|l|}{ Cor } \\
\hline & Branca & 48,8 & 54,1 & 45,9 & 0,1415 & 17,3 & 82,7 & 0,1839 \\
\hline & Não Branca & 51,2 & 60,8 & 39,2 & & 22,4 & 77,6 & \\
\hline \multicolumn{9}{|c|}{ Socioeconômicas } \\
\hline \multicolumn{9}{|c|}{ Escolaridade } \\
\hline & Analf/NS/NQR & 4,9 & 58,9 & 41,1 & 0,3408 & 15,2 & 84,8 & 0,0924 \\
\hline & Fundamental & 65,6 & 57,9 & 42,1 & & 22,8 & 77,2 & \\
\hline & Médio & 16,1 & 62,2 & 37,8 & & 14,3 & 85,7 & \\
\hline & Superior ou Mais & 13,4 & 49,5 & 50,5 & & 14,0 & 86,0 & \\
\hline \multicolumn{9}{|c|}{ Plano de saúde } \\
\hline & Sim & 51,4 & 57,0 & 43,0 & 0,8086 & 21,8 & 78,2 & 0,3273 \\
\hline & Não & 48,6 & 58,1 & 41,9 & & 17,9 & 82,1 & \\
\hline \multicolumn{9}{|l|}{ Trabalha } \\
\hline & Sim & 24,8 & 57,5 & 42,5 & 0,9938 & 23,6 & 76,4 & 0,3288 \\
\hline & Não & 75,2 & 57,5 & 42,5 & & 18,7 & 81,3 & \\
\hline \multicolumn{9}{|c|}{ Comportamentais } \\
\hline \multicolumn{9}{|l|}{ Hortaliça } \\
\hline & Sim & 57,1 & 58,0 & 42,0 & 0,8094 & 17,5 & 82,5 & 0,1707 \\
\hline & Não & 42,9 & 56,9 & 43,1 & & 23,1 & 76,9 & \\
\hline \multicolumn{9}{|l|}{ Frutas } \\
\hline & $\operatorname{Sim}$ & 73,1 & 58,5 & 41,5 & 0,4988 & 19,9 & 80,1 & 0,9798 \\
\hline & Não & 26,9 & 54,8 & 45,2 & & 19,8 & 80,2 & \\
\hline \multicolumn{9}{|c|}{ Hortaliça/Frutas } \\
\hline & Sim & 46,2 & 58,6 & 41,4 & 0,6727 & 17,7 & 82,3 & 0,2948 \\
\hline & Não & 53,8 & 56,6 & 43,4 & & 21,8 & 78,2 & \\
\hline \multicolumn{9}{|c|}{ Consumo de Doces } \\
\hline & Sim & 15,4 & 48,5 & 51,5 & 0,0819 & 20,8 & 79,2 & 0,8394 \\
\hline & Não & 84,6 & 59,1 & 40,9 & & 19,7 & 80,3 & \\
\hline \multicolumn{9}{|l|}{ Lanche } \\
\hline & Sim & 33,8 & 66,1 & 33,9 & 0,0048 & 27,3 & 72,7 & 0,0084 \\
\hline & Não & 66,2 & 53,1 & 46,9 & & 16,2 & 83,8 & \\
\hline \multicolumn{9}{|l|}{ Leite } \\
\hline & Sim & 43,1 & 55,9 & 44,1 & 0,5424 & 15,4 & 84,6 & 0,0562 \\
\hline & Não & 56,9 & 58,7 & 41,3 & & 23,3 & 76,7 & \\
\hline Muito Sal & & & & & & & & \\
\hline
\end{tabular}




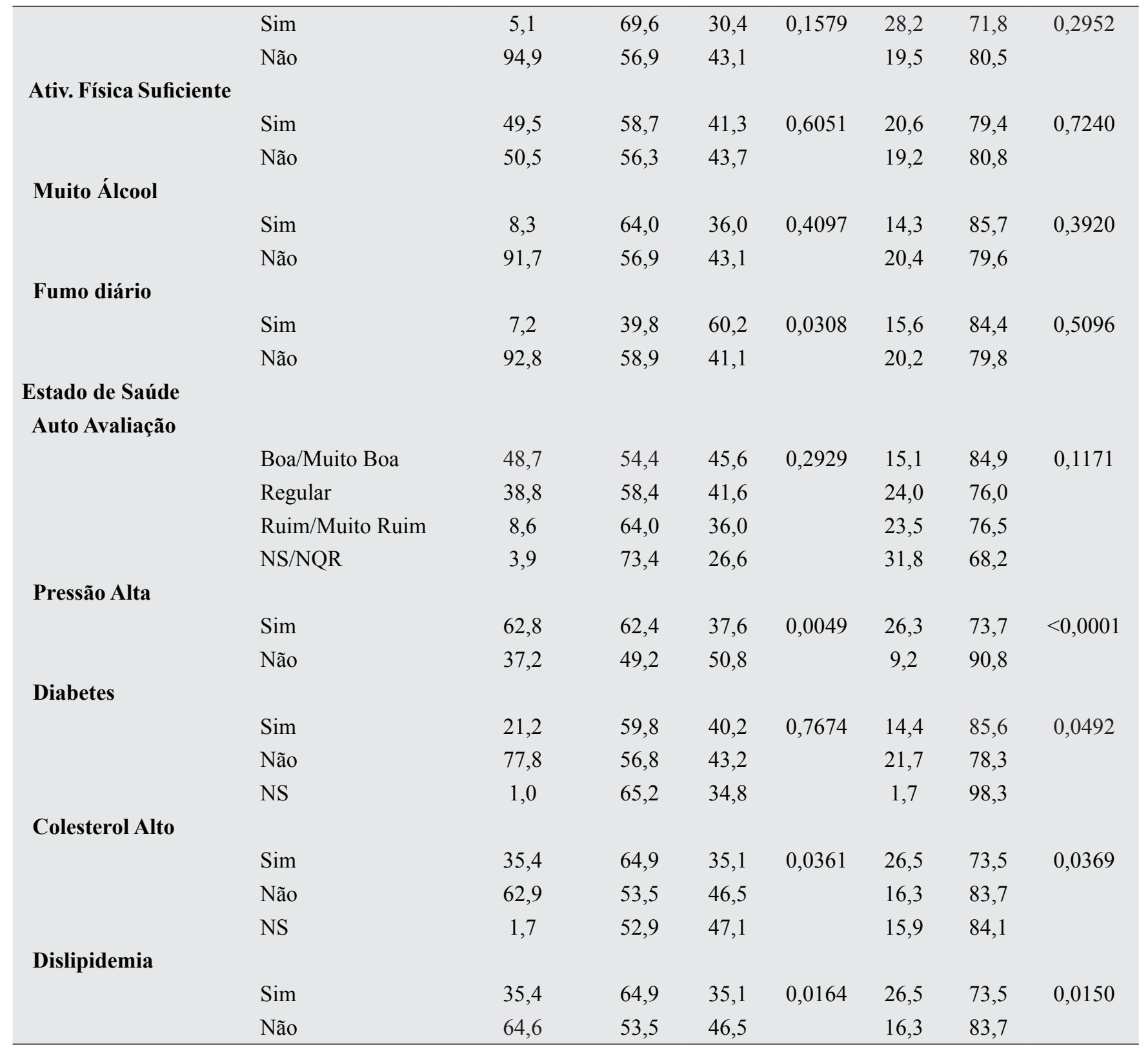

Legenda: Analf = Analfabeto; NS = Não sabe; NQR = Não quis responder. A tabela não apresenta os valores absolutos (n), por se tratar de uma amostra complexa e não retratar univocamente o número de pessoas observadas, diferente das porcentagens estimadas que são representativas ao nível populacional.

Dentre as variáveis significativas ao nível de $20 \%$ na análise bivariada (Tabela I), avaliou-se a possível existência de multicolinearidade. Dado que as variáveis dislipidemia e colesterol são altamente associadas, optou-se por colocar no modelo múltiplo a primeira, que apresentou maiores significâncias com os desfechos. A Tabela II apresenta os modelos ajustados com os melhores preditores para excesso de peso e para obesidade.

Observa-se que quem tem pressão alta, dislipidemia e hábito de substituir as refeições principais por lanche (cinco ou mais vezes na semana), tem maior chance de apresentar excesso de peso e obesidade. A probabilidade de ter excesso de peso diminui com o avançar da idade, assim como ser fumante diário (Tabela II). 
Tabela II - Modelos de regressão logística para fatores associados ao excesso de peso e à obesidade em idosos, Rio de Janeiro, VIGITEL 2013, Brasil.

\begin{tabular}{lcccc}
\hline Variável & \multicolumn{2}{c}{ Excesso de Peso } & \multicolumn{2}{c}{ Obesidade } \\
& OR & IC (90\%) & OR & IC (90\%) \\
\hline Pressão Alta & & & & \\
$\quad$ Sim & 1,665 & $1,132-2,447$ & 3,855 & $2,379-6,249$ \\
$\quad$ Lanche & 1,828 & $1,253-2,666$ & 2,181 & $1,432-3,323$ \\
$\quad$ Sim & & & & \\
$\quad$ Dislipidemia & 1,486 & $1,002-2,204$ & 1,816 & $1,202-2,743$ \\
$\quad$ Sim & & & & \\
$\quad$ Fumo diário & 0,446 & $0,211-0,943$ & - & - \\
$\quad$ Sim & 0,968 & $0,944-0,991$ & 0,938 & $0,913-0,964$ \\
$\quad$ Idade & & & 0,001 & $8,1 \mathrm{e}^{-6}-0,116$ \\
$\quad$ Diabetes & & & & \\
$\quad$ Sim & - & & 1,093 & $1,022-1,167$ \\
$\quad$ Termo de Interação \\
$\quad$ Idade X Diabetes (Sim)
\end{tabular}

* Foram adotados dois modelos e nem todas as variáveis explicativas permaneceram em ambos. Foi adicionado um traço à tabela, nos resultados ausentes.

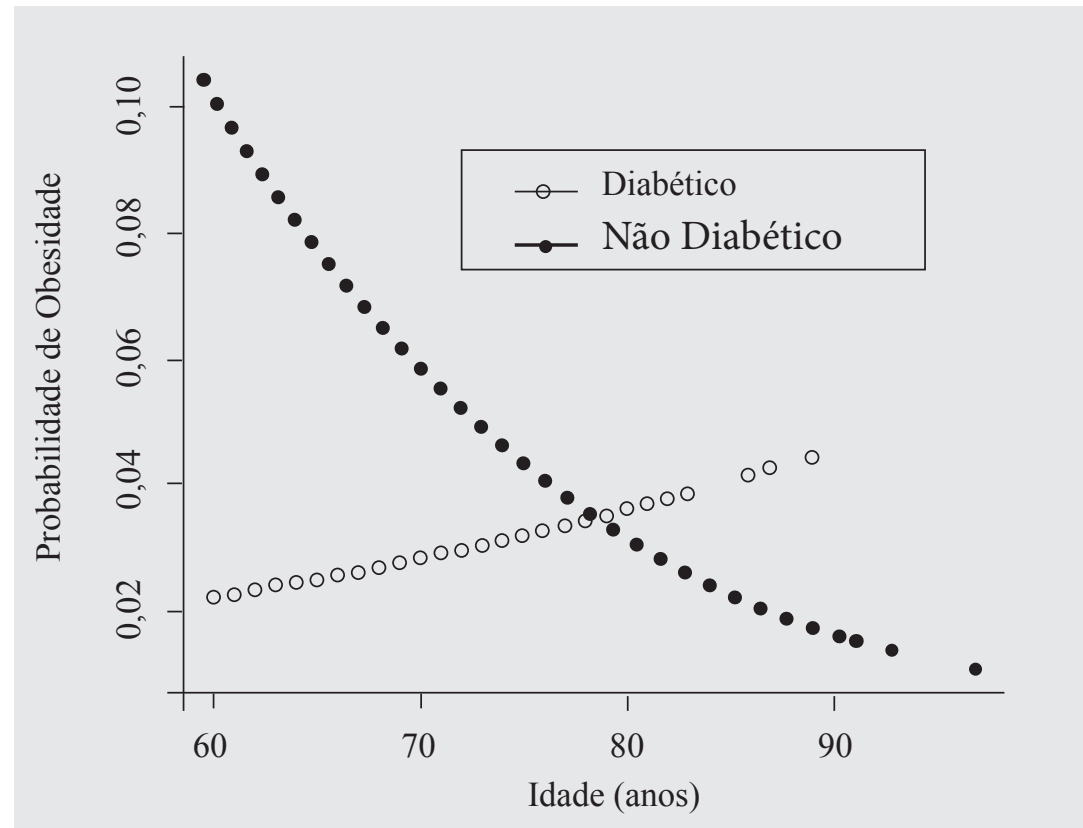

Figura 1 - Efeito da idade sobre a chance de ter obesidade, segundo a informação de ter ou não diabetes em idosos, Rio de Janeiro, VIGITEL, 2013, Brasil.

Além disso, existe associação entre diabetes e idade, indicando que a probabilidade de ser obeso aumenta com a idade entre diabéticos e diminui entre não diabéticos. Além disso, essa probabilidade é maior entre não diabéticos até os 79,5 anos de idade. A partir dessa idade, a probabilidade de ser obeso é maior entre diabéticos (Figura 1).

\section{DISCUSSÃO}

Entre os fatores com reconhecida associação com o desenvolvimento das DCNT, quatro foram observados na população avaliada no presente estudo: o tabagismo, o excesso de peso/obesidade, a hipertensão arterial e a dislipidemia. Os resultados indicam que a população idosa da cidade do Rio de Janeiro encontra-se com o estado nutricional insatisfatório, tendo sido observado percentual elevado de excesso de peso $(57,5 \%)$ e obesidade $(19,9 \%)$. No entanto, esses resultados são inferiores a outros estudos brasileiros ${ }^{(15,16)}$. 
Um estudo transversal de base populacional, com 596 idosos residentes em Pelotas, Rio Grande do Sul, encontrou prevalência de obesidade de 48,7\% (IC95\%: 44,6-52,7) ${ }^{(15)}$. Outro estudo transversal de base populacional, com 304 idosos do município de Porto Alegre, Rio Grande do Sul, apresentou prevalência de obesidade de 30,6\% ${ }^{(16)}$. De acordo com os dados da Pesquisa de Orçamentos Familiares (POF) 2008-2009, a prevalência de excesso de peso em uma amostra nacional de 20.114 indivíduos acima de 60 anos de idade foi de 37,3\%, sendo inferior à do presente estudo ${ }^{(17)}$.

A obesidade é uma doença crônica interrelacionada com outras enfermidades, como doenças cardiovasculares e câncer. Estratégias referentes à prevenção e ao tratamento do sobrepeso/obesidade junto à população idosa brasileira devem ser estabelecidas com o objetivo de minimizar os agravos acarretados pelo excesso de peso ${ }^{(18,19)}$.

Observou-se, na presente pesquisa, que a chance de desenvolvimento de obesidade diminui com o avanço da idade. $\mathrm{O}$ aumento da massa corporal inicia-se por volta dos 45 a 50 anos, estabilizando-se aos 70, quando começa a declinar até os $80 \operatorname{anos}^{(20)}$. Essa perda de peso é resultado de múltiplos fatores que envolvem o idoso, tais como: a dependência funcional nas atividades da vida diária; o uso excessivo de medicamentos; a depressão e o isolamento; as dificuldades financeiras; as alterações na dentição; o alcoolismo; o sedentarismo e, principalmente, a atrofia muscular e catabolismo associado às doenças agudas ou crônicas ${ }^{(21)}$.

Estimar a prevalência da diabetes mellitus por morbidade referida é vantajoso, pois é uma forma rápida e de baixo custo para obter a informação, o que viabiliza sua utilização em grandes populações ${ }^{(22,23)}$. A prevalência detectada no presente estudo $(21,2 \%)$ revelou-se superior à do estudo transversal de base populacional e domiciliar realizado em Campina Grande, Paraíba, no qual a prevalência de diabetes referida foi de $14,7 \%$ no conjunto de 806 idosos residentes e esteve significativamente associada ao excesso de peso nas idosas (OR: 2,09; IC95\%: 1,03-4,25). O excesso de tecido adiposo é um dos principais fatores para a ativação de vias bioquímicas inflamatórias, que causam prejuízos na sinalização intracelular da insulina ${ }^{(24)}$.

$\mathrm{Na}$ presente pesquisa, encontrou-se associação positiva entre as dislipidemias, HAS (hipertensão arterial sistêmica) e excesso de peso ou obesidade. Dados provenientes de um estudo transversal de base populacional apontaram uma associação entre dislipidemia e excesso de peso, revelando uma prevalência de $51,4 \%$ de dislipidemia nos idosos residentes do município de São Paulo sem excesso de peso, e 69,9\% naqueles com excesso de peso (p-valor: 0,01) ${ }^{(25)}$. Cerca de 250 idosos em tratamento ambulatorial em um Centro de Saúde de Portugal foram avaliados, tendo sido identificado 43,6\% da amostra com sobrepeso e $39,6 \%$ com obesidade. Metade das pessoas com 65 ou mais anos que apresentam sobrepeso e obesidade têm HAS, indicando que muitos idosos hipertensos têm peso corporal excessivo ${ }^{(26)}$.

A HAS autorreferida é um indicador apropriado para vigilância da prevalência da hipertensão arterial, na ausência da pressão arterial medida, sendo associada positivamente ao excesso de peso ${ }^{(27)}$. Cerca de $70 \%$ da população avaliada no presente estudo referiu diagnóstico médico de hipertensão arterial. Alterações próprias do envelhecimento tornam o indivíduo mais propenso ao desenvolvimento de HAS, o que se revela preocupante por representar uma das maiores causas de redução da qualidade e expectativa de vida dos indivíduos ${ }^{(28)}$.

A baixa ingestão de frutas e hortaliças está entre os principais fatores etiológicos para o excesso de peso ${ }^{(29)}$. Entretanto, no presente estudo, verificou-se alto percentual de idosos que as consomem regularmente. Um estudo que analisou dados do VIGITEL de 2012 revelou que a ingestão regular de frutas e hortaliças tende a aumentar com a idade (37,7\% na faixa de 45 a 54 anos; $42,1 \%$ dos 55 aos 64 anos; e 46,2\% na faixa de 65 ou mais anos), contudo, ainda é muito inferior ao mínimo de 400 g/dia, valor recomendado pela Organização Mundial da Saúde ${ }^{(30)}$. Para manutenção de um organismo saudável, o Guia Alimentar para a População Brasileira recomenda que sejam consumidos mais alimentos in natura em detrimento aos alimentos ultraprocessados ${ }^{(31)}$.

Menos da metade dos avaliados na presente investigação fazia ingestão diária de leite, cujo consumo é recomendado por ser a principal fonte de cálcio da dieta. A baixa ingestão desse nutriente leva ao aumento dos níveis sanguíneos de paratormônio e vitamina $\mathrm{D}$, que estimulam a atividade de enzimas relacionadas à lipogênese e inibem a lipólise. O baixo consumo de cálcio interfere no nível desse mineral no interior das células do tecido adiposo, favorecendo as vias metabólicas envolvidas no aumento da gordura corporal ${ }^{(32)}$. Portanto, estratégias que incentivem a ingestão adequada de leite, frutas e hortaliças entre os idosos devem ser desenvolvidas como forma de melhorar a qualidade de vida e prevenir enfermidades ${ }^{(33)}$.

A alimentação desempenha um papel de destaque dentre os fatores etiológicos responsáveis por doenças crônicas, dentre elas, as doenças cardiovasculares, que constituem a principal causa de morbimortalidade em idosos. Hábitos alimentares saudáveis e fatores associados à prevenção de cardiopatias foram identificados em 212 idosos com HAS atendidos em uma unidade de saúde da família, em município do interior do estado do Rio Grande do Sul ${ }^{(34)}$. Encontrou-se consumo elevado de temperos industrializados e embutidos ricos em sódio, além de referência a se fazer menos de cinco refeições por dia. Estes achados reforçam a necessidade de orientações nutricionais direcionadas a essa população, uma vez que a falta de conhecimento e informação sobre a composição nutricional dos alimentos também pode determinar excesso no consumo ${ }^{(34)}$.

O presente estudo encontrou um pequeno percentual de idosos com consumo alto ou muito alto de sal (5,1\%). Esse dado contraria um estudo anterior cujos resultados mostram que o consumo de sal no Brasil é alto, excedendo os limites máximos recomendados para a sua ingestão em todas as regiões do País e em todos os estratos de renda ${ }^{(35)}$. Por outro lado, a alta 
prevalência de HAS encontrada nessa amostra e o reconhecimento pelos idosos da gravidade desta doença provocada pela ingestão excessiva de sal pode ter sido responsável pelo baixo consumo desse alimento.

Uma limitação do estudo em questão é a amostra do VIGITEL ter sido extraída do cadastro das linhas telefônicas residenciais existentes na cidade. A cobertura dessa rede não é universal, podendo ser particularmente baixa em estratos de menor nível socioeconômico, o que se procurou contornar pela aplicação de pesos pós-estratificação aos entrevistados ${ }^{(9)}$. Outro aspecto a se considerar seria o possível viés de sobrevivência, que tende a reduzir a magnitude das associações encontradas entre fatores de risco e doença, uma vez que idosos expostos a fatores de risco têm maior probabilidade de morte prematura ${ }^{(36)}$. Essa é uma possível explicação para a associação entre obesidade e idade nos diabéticos: a maior probabilidade de ser obeso nos diabéticos com 80 ou mais anos de idade pode ser decorrente de já terem falecido aqueles que não tiveram o devido cuidado com a doença.

Como força do presente estudo, pode-se mencionar a análise de dados coletados pelo VIGITEL de idosos residentes no Rio de Janeiro, em 2013. Conhecer e divulgar o perfil dos idosos com excesso de peso e obesidade dessa cidade materializa o objetivo do sistema do monitoramento da frequência e distribuição de fatores de risco e proteção para as DCNT.

\section{CONCLUSÃO}

Uma parcela importante dos idosos residentes na cidade do Rio de janeiro apresenta um estado nutricional insatisfatório representado por um elevado percentual de excesso de peso e obesidade. A associação positiva observada entre hipertensão, dislipidemia e hábito de substituir as refeições principais por lanche (cinco ou mais vezes na semana), com os desfechos excesso de peso e obesidade, indicam a necessidade de adoção de medidas que estimulem o hábito alimentar saudável, assim como a manutenção de peso em faixa considerada segura para a prevenção de agravos.

\section{CONFLITOS DE INTERESSES}

Os autores declaram a inexistência de conflitos de interesse no presente estudo.

\section{REFERÊNCIAS}

1. Closs VE, Schwanke CHA. A evolução do índice de envelhecimento no Brasil, nas suas regiões e unidades federativas no período de 1970 a 2010. Rev Bras Geriatr Gerontol. 2012;15(3):443-58.

2. Instituto Brasileiro de Geografia e Estatística (IBGE). Censo de 2010 [acesso em 2016 Jan 10]. Disponível em: https:// censo2010.ibge.gov.br/

3. Bezerra FC, Almeida MI, Nóbrega-Therrien SM. estudos sobre envelhecimento no brasil: revisão bibliográfica. Rev. Bras. Geriatr. Gerontol., 2012;15(1):155-67.

4. Silva JVF, Silva EC, Rodrigues PARA, Miyazawa AP. A relação entre o envelhecimento populacional e as doenças crônicas não transmissíveis: sério desafio de saúde pública. Cadernos Graduação Ciênc Biol Saúde. 2015;2(3):91-100.

5. Venturini CD, Engroff P, Sgnaolin V, El Kik RM, Morrone FB, Silva IG Filho, De Carli GA. Consumo de nutrientes em idosos residentes em Porto Alegre (RS), Brasil: um estudo de base populacional. Ciênc Saúde Colet. 2015;20(12):3701-11.

6. Ferreira SAD. Potencialidade dos inquéritos em saúde: uma contribuição para a epidemiologia nutricional [tese]. Belo Horizonte: Universidade Federal de Minas Gerais; 2011.

7. Mássimo EAL, Souza HNF, Freitas MIF. Doenças crônicas não transmissíveis, risco e promoção da saúde: construções sociais de participantes do Vigitel. Ciênc Saúde Colet. 2015;20(3):679-88.

8. Malta DC, Iser BPM, Claro RM, Moura L, Bernal RTI, Nascimento AF, et al. Prevalência de fatores de risco e proteção para doenças crônicas não transmissíveis em adultos: estudo transversal, Brasil, 2011. Epidemiol Serv Saúde. 2013;22(3):42334.

9. Ministério da Saúde (BR). VIGITEL - Vigilância de Fatores de Risco e Proteção para Doenças Crônicas por Inquérito Telefônico. Estimativas sobre frequência e distribuição sociodemográfica de fatores de risco e proteção para doenças crônicas nas capitais dos 26 estados brasileiros e do Distrito Federal em 2013. Brasília: Ministério da Saúde; 2014.

10. Ainsworth BE, Haskell WL, Herrmann SD, Meckes N, Bassett DR Jr, Tudor-Locke C, et al. Compendium of physical activity: second update of codes and MET values. Med Sci Sports Exerc. 2011;43(8):1575-81.

11. Rao JNK, Scott AJ. On chi-squared tests for multiway contingency-tables with cell proportions estimated from survey data. Ann Stat. 1984;12:46-60. 
12. Francisco PMSB, Donalisio MR, Barros MBA, Cesar CLG, Carandina L, Goldbaum M. Medidas de associação em estudo transversal com delineamento complexo: razão de chances e razão de prevalência. Rev Bras Epidemiol. 2008;11(3):34755.

13. Dobson AJ. An introduction to generalized linear models. $2^{\text {nd }}$ ed. London: Chapman and Hall/CRC; 2001.

14. R Development Core Team. R: A language and environment for statistical computing. Vienna: R Foundation for Statistical Computing; 2010 [acesso em 2017 Mar 5]. Disponível em: http://www.R-project.org

15. Costa CS, Schneider BC, Cesar JA. Obesidade geral e abdominal em idosos do Sul do Brasil: resultados do estudo COMO VAI? Ciênc Saúde Colet. 2016;21(11):3585-96.

16. Venturini CD, Engroff $\mathrm{P}$, Gomes I, De Carli GA. Prevalência de obesidade associada à ingestão calórica, glicemia e perfil lipídico em uma amostra populacional de idosos do Sul do Brasil. Rev Bras Geriatr Gerontol, 2013;16(3):591-601.

17. Pereira IFS, Spyrides MHC, Andrade LMB. Estado nutricional de idosos no Brasil: uma abordagem multinível. Cad Saúde Pública, 2016;32(5):e001788142016.

18. Ferreira PM, Rosado GP. Perfil de usuários e percepção sobre a qualidade do atendimento nutricional em um programa de saúde para a terceira idade. Rev Bras Geriatr Gerontol., 2012;15(2):243-54.

19. Santos RR, Bicalho MAC, Mota P, Oliveira DR, Moraes EN. Obesidade em idosos. Rev Med Minas Gerais. 2013;23(1):6271.

20. Matsudo SM, Matsudo VKR, Barros TL Neto. Impacto do envelhecimento nas variáveis antropométricas, neuromotoras e metabólicas da aptidão física. Rev Bras Ciênc Mov. 2000;8(4):21-32.

21. Almeida RSV, Melo TGC, Pereira IFM, Cabido CET. Treinamento de força e desempenho do sistema neuromuscular em idosos. e-Scientia. 2014;7(1):16-26.

22. Pimenta FB, Pinho L, Silveira MF, Botelho ACC. Fatores associados a doenças crônicas em idosos atendidos pela Estratégia de Saúde da Família. Ciênc Saúde Colet. 2015;20(8):2489-98.

23. Menezes TN, Sousa NDS, Moreira AS, Pedraza DF. Diabetes mellitus referido e fatores associados em idosos residentes em Campina Grande, Paraíba. Rev Bras Geriatr Gerontol. 2014; 17(4):829-83.

24. Freitas MC, Ceschini L, Ramallo BT. Resistência à insulina associado à obesidade: efeitos anti-inflamatórios do exercício físico. Rev Bras Ciênc Mov. 2014;22(3):139-47.

25. Garcez MR, Pereira JL, Fontanelli MM, Marchioni DML, Fisberg RM. Prevalência de dislipidemia segundo estado nutricional em amostra representativa de São Paulo. Arq Bras Cardiol. 2014;103(6):476-84.

26. Mártires MAR, Costa MAM, Santos CSV. Obesidade em idosos com hipertensão arterial sistémica. Texto \& Contexto Enferm. 2013;22(3):797-803.

27. Selem SSC, Castro MA, César CLG, Marchioni DML, Fisberg RM. Validade da hipertensão autorreferida associa-se inversamente com escolaridade em brasileiros. Arq Bras Cardiol. 2012;100(1):52-9.

28. Oliveira JG Neto, Carvalho DA, Sá GGM, Monteiro MM, Lopes KDCL, Martins MCC. Pressão arterial e perfil socioeconômico de idosos atendidos na Estratégia Saúde da Família de Floriano-PI. Rev Saúde Pública. 2014;7(2):17-28.

29. Machado RHV, Feferbaum R, Leone C. Consumo de frutas no Brasil e prevalência de obesidade. Rev Bras Crescimento Desenvolv Hum. 2016;26(2):243-52.

30. Malta DC, Bernal RTI, Nunes ML, Oliveira MM, Iser BPM, Andrade SSCA, et al. Prevalência de fatores de risco e proteção para doenças crônicas não transmissíveis em adultos: estudo transversal, Brasil 2012. Epidemiol Serv Saúde. 2014;23(4):609-22.

31. Ministério da Saúde (BR), Secretaria de Atenção à Saúde, Departamento de Atenção Básica, Coordenação Geral de Alimentação e Nutrição. Guia alimentar para a população brasileira: promovendo a alimentação saudável. Brasília: Ministério da Saúde; 2014.

32. Arruda AP, Hotamisligil GS. calcium homeostasis and organelle function in the pathogenesis of obesity and diabetes. Cellular Metabolism. 2015;22(3):381-97.

33. Deon RG, Rosab RD, Zanardoc VPS, Clossd VE, Schwanke CHA. Consumo de alimentos dos grupos que compõem a pirâmide alimentar americana por idosos brasileiros: uma revisão. Ciênc Saúde Colet. 2015;8(1):26-34. 
34. Gadenz SD, Benvegnú LA. Hábitos alimentares na prevenção de doenças cardiovasculares e fatores associados em idosos hipertensos. Ciênc Saúde Colet. 2013;18(12):3523-33.

35. Sarno F, Claro RM, Levy RB, Bandoni DH, Ferreira SRG, Monteiro CA. Estimativa de consumo de sódio pela população brasileira, 2008-2009. Rev Saúde Pública. 2013;47(3):571-8.

36. Lima-Costa MF, Barreto SM. Tipos de estudos epidemiológicos: conceitos básicos e aplicações na área do envelhecimento. Epidemiol Serv Saúde. 2003;12(4):189-201.

\section{Endereço para correspondência:}

Celia Cristina Diogo Ferreira

Universidade Federal do Rio de Janeiro - UFRJ

Avenida Aloizio da Silva Gomes, 50

Bairro: Novos Cavaleiros

CEP: 27930-560 - Macaé - RJ - Brasil

E-mail: celia.ferreira@macae.ufrj.br 